\title{
Deep learning-based computational drug discovery to inhibit the RNA Dependent RNA Polymerase: application to SARS-CoV and COVID-19
}

\author{
Sayalee Patankar* \\ Adlai E. Stevenson High School, Lincolnshire, IL 60069 \\ *Email: spatank21@students.d125.org
}

\begin{abstract}
There is an urgency to find drugs and vaccines for the 2019 coronavirus disease (COVID19). Therapeutic options include repurposing existing drugs or finding new ones. One approach is to target the RNA-dependent RNA polymerase (RdRp) and block viral RNA synthesis (Li et. al., 2020). Currently clinical trials to repurpose remdesivir, a RdRp targeting pro-drug for Ebola, to COVID-19 is under way ( $\mathrm{Li}$ et. al., 2020). More such potential drugs need to be identified to efficiently find best therapeutic options. To address this need, a Long Short Term Memory (LSTM) model from literature (Bjerrum, 2017) was trained to read the SMILES fingerprint of a molecule and predict the IC50 of the molecule when binding to an RdRp. This model was trained using IC50 binding data from the PDB database. 310,000 drug-like compounds from the ZINC database were then screened using the trained LSTM model. Additionally, the 310,000 molecules with their predicted IC50s were used to train a generative Semi-Supervised Variational AutoEncoder (SSVAE) model from literature (Kang et. al., 2019). Although not trained by actual experimental data (sufficient data are not available), the SSVAE model was used to generate 10 new molecules by sampling from the latent space to demonstrate its utility. These 10 molecules and the 1025 molecules with the lowest predicted IC50s from the LSTM model were docked onto the SARS coronavirus (a virus similar to COVID-19) RdRp using AutoDock Vina. Top four most stable inhibitors from the screened ZINC database compounds had binding energies of less than -33.89 $\mathrm{kJ} / \mathrm{mol}$. These binding energies were less than the binding energies of the comparison group consisting of prior drugs remdesivir, favipiravir, and galidesivir. Among the ten new molecules generated by the SSVAE model, the most stable new molecule had binding energy lower than the comparison group of prior drugs. The low binding energies of these molecules indicate they could potentially be good drug candidates for the SARS CoV and COVID-19. These results also show the utility of deep learning-based models in screening existing compound and generating new molecules to find drugs for COVID-19.
\end{abstract}




\section{Introduction}

The purpose of this work is to find compounds, using deep learning-based models, that will bind to the RNA dependent RNA polymerase (RdRp) of coronaviruses and prevent further viral replication. This work will use a computational method to generate and dock compounds onto the SARS coronavirus RdRp and determine which compounds will be better and more stable inhibitors, indicated by a lower IC50 and binding energy. The RNA dependent RNA polymerase (RdRp), the protein targeted in this study, is present in all RNA viruses that lack a DNA stage. This includes the Zika virus, Ebola virus, Nipah virus, coronaviruses, and many more. This work will focus specifically on the SARS (severe acute respiratory illness syndrome) coronavirus (SARS-CoV) in hopes of finding drugs that can inhibit the novel 2019 coronavirus (COVID-19). This approach is used because the protein structures of COVID-19 are still being found. The COVID-19 has a genome that is over $82 \%$ identical to that of SARS-CoV. Therefore, compounds that can inhibit SARS-CoV potentially have a good chance of also inhibiting COVID-19.

The proposed compounds can also bring us one step closer to finding a drug that can inhibit the growth of RNA viruses in general. RNA viruses can cause a variety of symptoms, which can sometimes be life threatening. The Zika virus, for example, is suspected to cause microcephaly in babies if the woman is infected during pregnancy (Centers for Disease Control and Prevention, n.d.). Microcephaly is when a baby's head is smaller than expected. Usually if a baby has microcephaly, they have smaller brains that have not completely grown or developed. More serious, and often deadly, the Ebola virus can cause hemorrhaging (bleeding) (Centers For Disease Control and Prevention, 2019) and the Nipah virus can cause encephalitis (swelling of the brain) (Centers For Disease Control and Prevention, March 2014). Finally, cornoviruses such as SARS (severe acute respiratory illness syndrome), MERS (middle east respiratory syndrome), and the recent COVID-19 can cause acute respiratory illness and death.

The RdRp is essential to the replication process of RNA viruses. The protein itself is highly conserved, meaning its structure is similar in all viruses that contain the enzyme. The enzyme is used to replicate the viral RNA genome of RNA viruses. It catalyzes the formation of phosphodiester bonds between ribonucleotides, the basic unit of RNA, to form a daughter strand of RNA that is nearly identical to the RNA template. The core of the enzyme has three domains: the thumb, palm, and fingers. These domains create a cup-like shape in active site, which perform numerous functions during the reaction. Some include binding to the template RNA strand, binding 
to incoming nucleoside triphosphates, aiding in the formation of the phosphodiester bond between the incoming nucleotide and the daughter RNA strand, and translocating the template strand to repeat the process (Venkataraman et. al., 2018).

Because ribonucleoside trisphosphates naturally bind to the polymerase, their structure is often mimicked to create drugs that will bind to and block the active site of the polymerase, thus inhibiting its function and further replication of the virus. A ribonucleoside triphosphate consists of the five carbon sugar ribose, three phosphate groups, and a nitrogenous base. There are four RNA nitrogenous bases: adenine, uracil, guanine, and cytosine (Wan et. al., 2018).

Favipiravir, galidesivir, and remdesivir are three compounds that have shown inhibitory function of the RdRp ( $\mathrm{Li}$ et. al., 2020). Favipiravir is proven to treat an influenza in Japan. Galidesivir has been shown to bind to the RdRp of Hepatisis C. Finally, Remdesivir has demonstrated inhibitory functions of the Ebola virus. All three of these compounds are prodrugs, meaning they become functional after being activated within the cell. Because the three molecules have proved to inhibit certain RdRps, they will be used as comparison groups for this study. The active forms of these compounds were obtained from Tchesnokov et. al., 2019 and will be used in the dockings.

In addition to these three compounds, there are many ligands in the Protein Data Bank (PDB) that are known to bind to a RdRp. Those that are also labelled with a corresponding half maximal inhibitory concentration (IC50) will be used to train the deep learning models described below. Finally, the ZINC database will be used as the experimental groups. This database consists of millions of commercially available compounds (Sterling et.al., 2015). A set of 310,000 druglike compounds from this database will be used in this study to find which of these drugs might potentially bind to RdRp.

\section{Materials and Methods}

2.1 Deep Learning Models Two types of deep learning models will be used in this study - a predictive model and a generative model. In the predictive model, a compound's Simplified Molecular-Input Line-Entry System (SMILES) fingerprint will be input into the model proposed earlier (Bjerrum, 2017). The SMILES fingerprint is simply a one-line string that codes for the molecule. The model will then be trained to predict the compound's binding property when binding to an RdRp. The binding property that will be used in this study is the IC50. The IC50 is the 
concentration of the inhibitor needed to reduce the enzyme's activity by half (GraphPad, 2010). Therefore, low IC50s are indicators of better inhibitors.

The input to the models must be arrays. Therefore, the SMILES strings must first be converted to a "one-hot-vector" to pass through the model. In the one-hot vector, each column represents an index in the SMILES sequence while each row is a specific character. Within a column, every value is 0 except for one location with value 1 . The 1 value indicates the presence of the character corresponding to its row while the 0s indicate no presence (Vasudev, 2019).

In addition to vectorizing the SMILES sequences, an enumeration of the SMILES sequences must be performed on the training data (Bjerrum, 2017). The enumeration is a form of data augmentation, which will increase the amount of training data. This step is important because the available data is not enough on its own to train an accurate model. Because more than one SMILES fingerprint can be produced for the same compound, the enumerator can generate multiple data points out of a single one by producing many fingerprints for the same compound (Bjerrum, 2017).

Once the arrays are made, they can then pass through the predictive model, which is made of many layers, each performing a function on the input arrays. Though there are many types of layers, only two types will be used in this model. The first is a type of recurrent neural network (RNN). The specific type of RNN that will be used (Bjerrum, 2017) is the Long Short Term Memory network (LSTM). This type of RNN allows the model to "remember" earlier information better, allowing it to make more accurate predictions (Olah, 2015). The next type of layer added after the LSTM layer is a dense layer (Bjerrum, 2017).

In order to train the model, molecules from the PDB database that are known to bind to an RdRp and are labelled with an IC50 will be used. Before this data can be used, however, it must be split into a training set and a testing set. About $75 \%$ of the data will be allotted for training and the remaining $25 \%$ will be used to test the accuracy of the model after training. The IC50 data will also be normalized so all the values are between -1 and 1 . This will help the model train better and be more accurate.

A second, more complex generative model that will be used in this study is the generative model (Kang et. al., 2019). This model is a Semi-Supervised Variational AutoEncoder (SSVAE). The model has three components: the predictor, the encoder, and the decoder. The predictor will read the SMILES sequence of a compound (x) and predict $\mathrm{y}$, which is the IC50 of the compound 
when binding to the RdRp. The encoder uses this information as well as the original SMILES sequence $\mathrm{x}$ to generate a low dimension latent vector $\mathrm{z}$. The vector $\mathrm{z}$ can then pass through the decoder to produce the reconstructed SMILES sequence x' (Kang et. al., 2019). This model is useful as it can be used to generate new molecules. One can sample from the latent space by choosing a certain $\mathrm{z}$ that is predicted to have a low IC50. This $\mathrm{z}$ can then be decoded into a SMILES string, yielding a new compound. This model uses (Kang et. al., 2019) Gated Recurrent Units (GRU). This layer is a type of RNN. It is similar to an LSTM except it has fewer parameters and is therefore quicker to train (Nguyen, 2019).

The models will be written with Python language in Google Colab, which is a free and paid GPU service (Fuat, 2019). It will use Keras, an open source deep learning library (Keras, n.d.), with the TensorFlow backend, a free machine learning platform (TensorFlow, n.d.).

2.2 Docking Model: AutoDock Vina AutoDock Vina allows its user to computationally predict the binding of a compound to a receptor with a known 3-dimensional structure. The result is multiple conformations of the ligand as well as their corresponding binding energies. The binding energy of a conformation is the difference between the energy of compound and protein in their unbound state and the energy of the compound protein complex (Raschka, 2014). This value must be negative for the binding to happen spontaneously and the lower this energy is, the more stable the compound will be and the better the compound is as a possible drug candidate.

The software includes AutoDock Tools, a graphical interface that allows for the visualization of the molecules and contains many features to prepare molecules for docking, such as the ability to add hydrogens and choose which bonds can be rotatable in the ligand (Morris, n.d.).

$\underline{\text { 2.3 Variables }}$ The relevant variables in this work are noted below after which the overall approach is summarized.

Independent Variable: Compounds from ZINC database that are screened through the LSTM deeplearning model, and new compounds generated from the SSVAE model.

Dependent Variable: IC50 (in deep learning models), Lowest binding energy (in AutoDock Vina). Constants:

- $\operatorname{RdRp}$ 
- RdRp search space (where the compound will be computationally docked)

- Docking process

- Softwares used in the procedure

Comparison Groups:

- Remdesivir (can bind to the RdRp in Ebola virus)

- Favipiravir (can bind to the RdRp in influenza virus)

- Galidesivir (can bind to the RdRp in Hepatitis C virus)

2.4 Approach A flowchart summarizing the approach of this work is shown in Figure 1. As noted above, in this project, a library of compounds will be proposed and computationally docked onto the SARS (Severe Acute Respiratory Syndrome) coronavirus RNA dependent RNA polymerase (RdRp) to find a potential drug for the novel 2019 coronavirus (COVID-19).

First, an LSTM deep learning model (Bjerrum, 2017) will be trained to read the SMILES fingerprint of a molecule and predict the IC50 of the molecule when binding to an RdRp (\#1 and \#2 in Figure 1). This model will be trained with data acquired from the PDB database (see Supplementary) and will consist of all compounds that are known to bind to an RdRp and have a known IC50. Because the model will be trained to predict the IC50 when binding to any RdRp, the model will be a useful tool for finding drugs to inhibit any virus that uses an RdRp to replicate. This includes most RNA viruses except retroviruses. 310,000 drug-like compounds (Bjerrum, 2017) from the ZINC database will be screened using the model to shortlist compounds for further testing (\#2 in Figure 1).

The 310,000 molecules along with their predicted IC50s will then be used to train a second deep learning model (\#3 in Figure 1). This model will be an SSVAE (Kang et. al., 2019). The model requires a large amount of data to train and this amount is not available through online databases. For this reason, the data generated by the LSTM model will be used to train the SSVAE. After training, the SSVAE will be used to generate 10 new molecules by sampling from the latent space.

The 10 molecules from the generative SSVAE model and 1025 molecules with the lowest predicted IC50s from the predictive LSTM model will be docked onto the SARS coronavirus RdRp (\#4 in Figure 1). This particular virus was selected because it is over $82 \%$ identical to the 
novel 2019 coronavirus and therefore compounds that are predicted to bind well to the SARS protein could also have a high probability of binding to the COVID-19 RdRp.

The selected compounds' SMILES fingerprints will be input into Avogadro to build 3D coordinates and convert the sequence to $\mathrm{a}$ pdb structure. PyRx will then be used to energy minimize the structures and convert to AutoDock Vina formats. The minimization of the structures brings the compounds to a low energy state in which they would be more likely be in reality. This provides a realistic starting conformation for the docking. AutoDock Vina can then be used to dock the compounds onto the SARS virus polymerase.

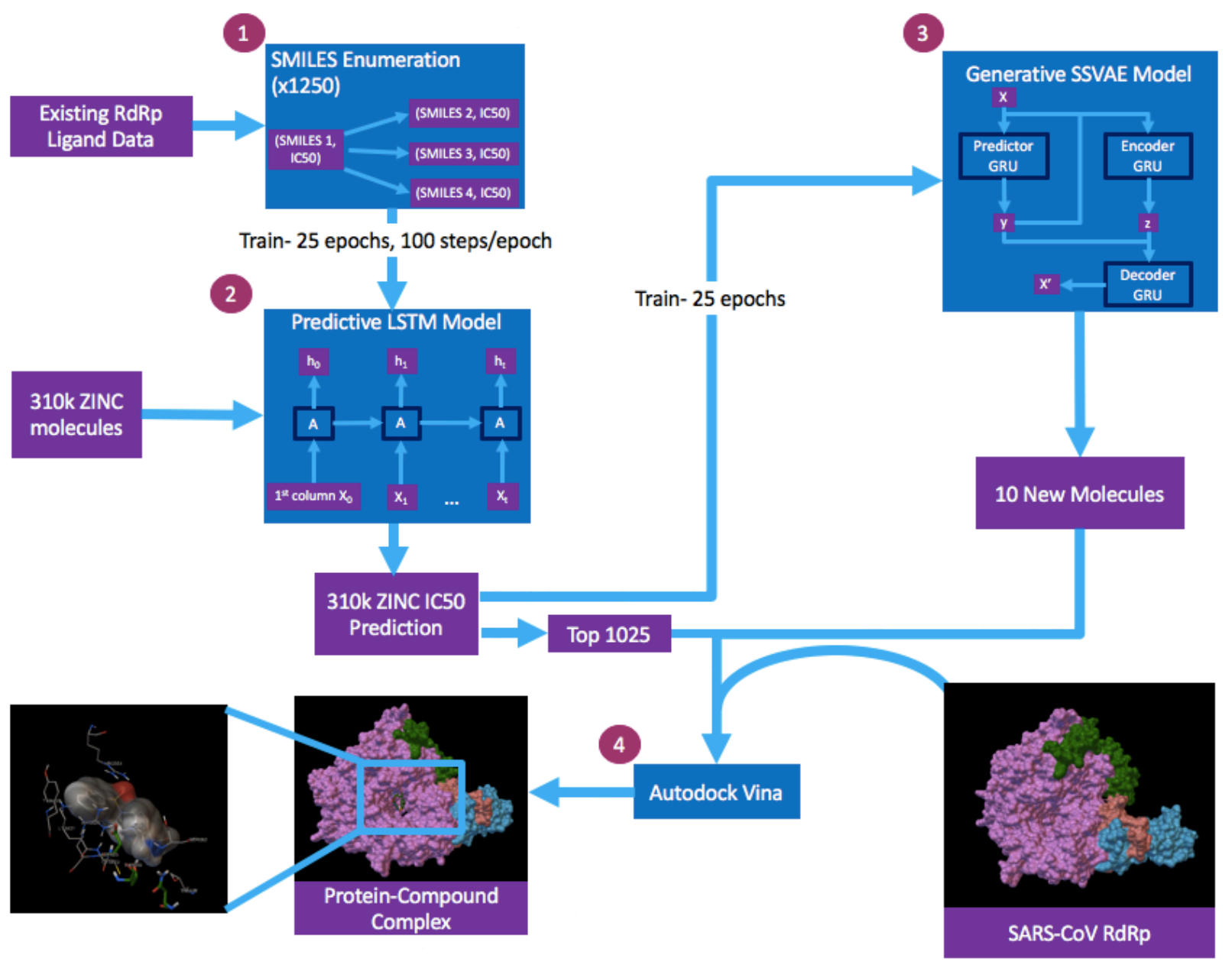

Figure 1 A flowchart summarizing the approach in this work. The SMILES enumeration and LSTM model is by Bjerrum, 2017. The SSVAE model is by Kang et. al., 2019. 


\section{$\underline{\text { 2.5 Materials }}$}

- A dual core 16 processors desktop computer

- Google Colab - used for deep learning model development and solution

- AutoDock Tools - to view molecules and edit them

- AutoDock Vina-docks compounds onto proteins to predict the binding conformation and corresponding energy

- Avogadro - to build 3D coordinates from a 2D file

- PyRx - to minimize the energy of a library of molecules and convert them into format that can be used in AutoDock Vina

- OpenBabel - to convert between file formats

- Access to the Protein Data Bank website

\section{Results and Discussion}

\section{$\underline{\text { 3.1 LSTM Deep Learning Model }}$}

The LSTM model (\#2 in Figure 1) was trained for 25 epochs with 100 steps per epoch using the RdRp ligand data from the PDB website (see Supplementary). Prior to training, each data point was enumerated (randomized and vectorized) 10 times, yielding 10 times as much data as the original. During training, each step further enumerated 210 of the SMILES sequences one time. Because two batches were needed to get through all the data, every data point was randomized 50 times for each epoch (1250 times total) during training. After the 25 epochs, the loss function began to converge at around 0.007. The loss used was the mean squared error, which is the average of the difference between estimated and actual values squared. In order to further validate that the model had properly trained, the testing and training sets were graphed with their actual values plotted along the $\mathrm{x}$ axis and their predicted values along the $\mathrm{y}$ axis (Figure 2). The resulting plot had a regression line with a slope equal to 0.9883 which is close to the ideal of one. The R squared value for the line was about 0.93 , suggesting a strong correlation. 


\section{Y Predict vs Y Actual}

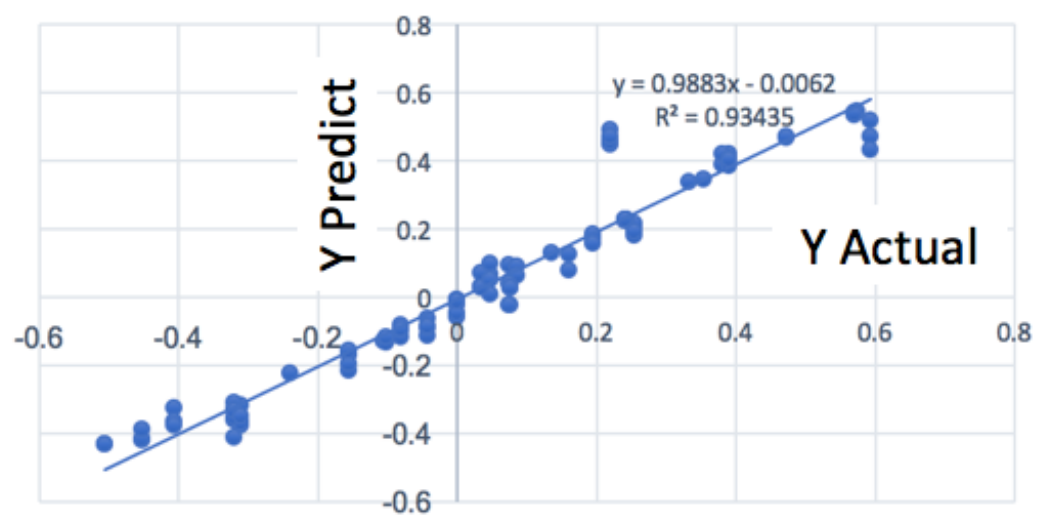

Figure 2 The graph shows the predicted IC50 (y predict) of the test data (a portion of the ZINC data) on the $y$ axis and the actual IC50 (y actual) on the $x$ axis. The IC50s were normalized leading all values to be between -1 and 1. A line of best fit was plotted (equation in the figure) and the $r$ squared value for the data was calculated.

After training, the 310,000 drug-like compounds from the ZINC database were passed through the LSTM model (Figure 3; see data in Supplementary). An IC50 of $100 \mathrm{nM}$ is an indicator of a good inhibitor. This value corresponds to a y value of about 0.19 from the machine learning model. 253369 out of the 310,000 had predicted values less than or equal to this value. 297405 experimental molecules had predicted values less than at least one of the three comparison groups. Favipiravir had the highest predicted value out of the three comparison groups with about 0.377 . Galidesivir had the lowest predicted value out of the comparison groups with a value of about 0.319 . The 1025 molecules chosen for further testing had predicted values less than or equal to -0.419 which is corresponds to an IC50 of less than $1.7 \mathrm{nM}$ (see data in Supplementary). This low IC50 indicates the molecules chosen for further testing potentially have a high chance of being inhibitors of an RdRp. 


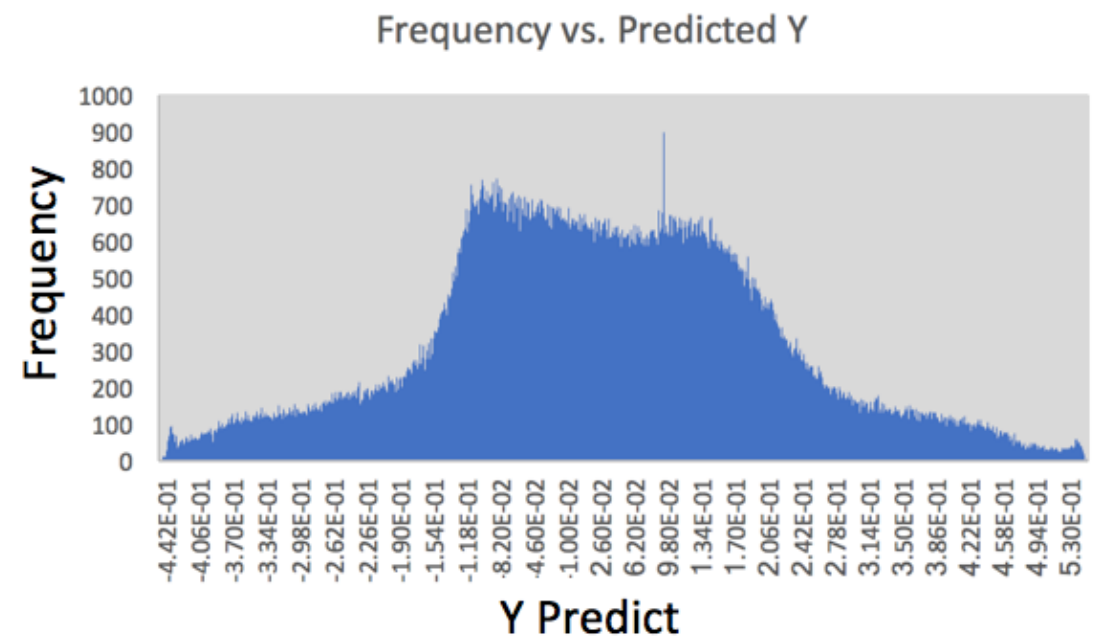

Figure 3 The graph shows the frequency of each predicted IC50 (predicted y) for the 310000 ZINC molecules after passing through the LSTM model. Molecules with a predicted y value of -0.419 or less were chosen for further testing with Autodock Vina. IC50s were normalized, leading all predicted $y$ values to be between -1 and 1 .

\subsection{SSVAE Deep Learning Model}

The SSVAE model (\#3 in Figure 1) was trained using the data generated for the ZINC molecules after passing through the LSTM model (see data in Supplementary). It was trained for 25 epochs after which the loss measures began to converge. 20 new molecules were generated using the SSVAE with a target y value of -0.4. This value corresponds to an IC50 of $1.7 \mathrm{nM}$ (the lowest IC50 in the PDB RdRp ligand data set). A lower value was not chosen because extrapolation can lead to inaccuracy. In order to verify the generated SMILES were not random, 16 unconditional SMILES sequences were also generated. These 16 were generated with no target y value and were instead 16 random molecules. The 20 conditionally generated molecules as well as the 16 unconditional molecules were input into the LSTM model (Figures 4 and 5). A greater frequency of the unconditional molecules $(75 \%)$ had positive y values which is associated with a higher IC50 and would be likely for random molecules. The conditional compounds had a greater frequency $(85 \%)$ of negative $\mathrm{y}$ values, indicating the generation had produced non-random molecules. Of the 20 conditionally generated molecules, 10 were docked onto the $\operatorname{RdRp}$ in Autodock Vina. 


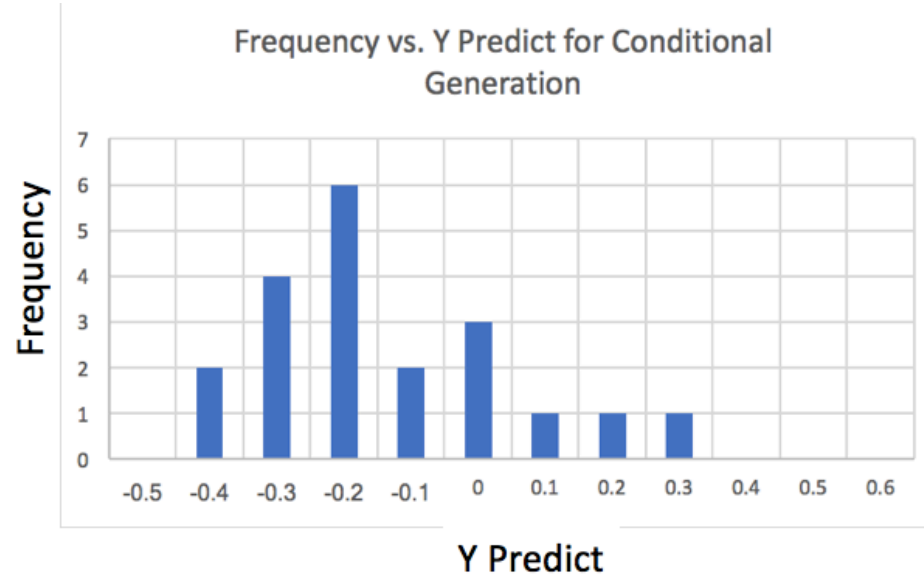

Figure 4 The graph shows the frequency of groups of predicted IC50s (predicted y) for the 20 conditional molecules after passing through the LSTM model. The 20 molecules were generated by the SSVAE with a target $y$ value of -0.4 (equivalent to an IC50 of $1.7 \mathrm{nM}$ ). The frequency at 0.4 is the number of molecules with $y$ values between -0.5 and -0.4 and so on. IC50s were normalized, leading all predicted $y$ values to be between -1 and 1 .

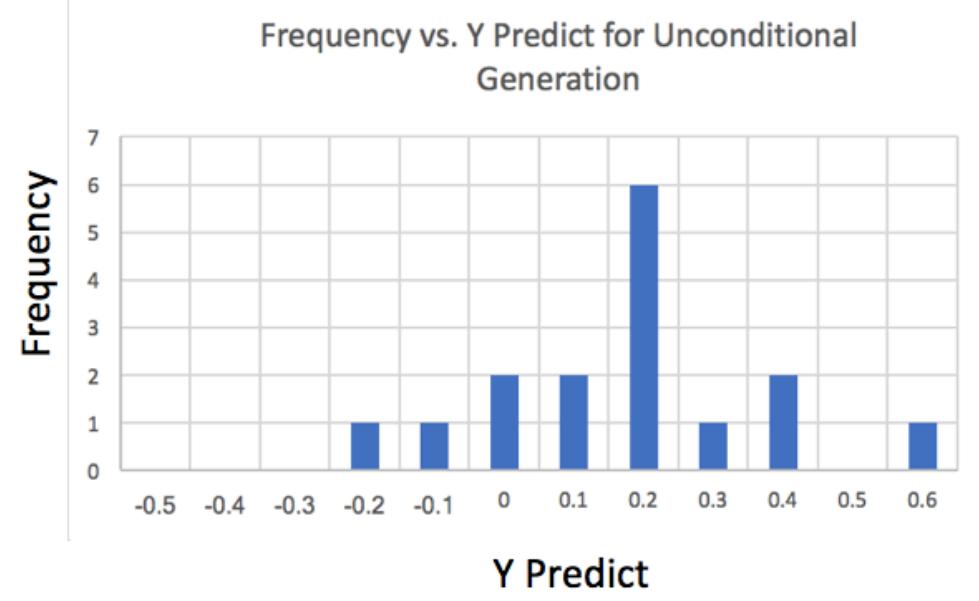

Figure 5 The graph shows the frequency of groups of predicted IC50s (predicted y) for the 16 unconditional molecules after passing through the LSTM model. The 16 molecules were generated by the SSVAE without a target $y$ value. The frequency at -0.2 is the number of molecules with $y$ values between -0.3 and -0.2 and so on. IC50s were normalized, leading all predicted $y$ values to be between -1 and 1 .

3.3 Autodock Vina Docking Top 1025 molecules from ZINC database that were screened through the LSTM model were docked in Autodock Vina to find their binding energies with RdRp of SARS coronavirus (see data in Supplementary). The results are shown in Figure 6. Similarly, 
top 10 newly generated molecules from SSVAE model were docked in Autodock Vina to find their binding energies with RdRp of SARS coronavirus (see data in Supplementary). The results are in Figure 7.

While the deep learning model results can apply to any viral RdRp, the dockings in Autodock Vina were specific to the RdRp from the SARS coronavirus. 1025 ZINC molecules, 10 conditionally generated molecules and the 3 comparison groups were docked and their lowest predicted binding energies were recorded. 600 out of the 1025 ZINC experimental molecules tested in Autodock Vina had binding energies less than at least one of the three comparison groups (less than $-25.5224 \mathrm{~kJ} / \mathrm{mol}$ ) (Figure 6). Of these 600, the top candidates could be compounds for the COVID-19 and SARS-CoV.

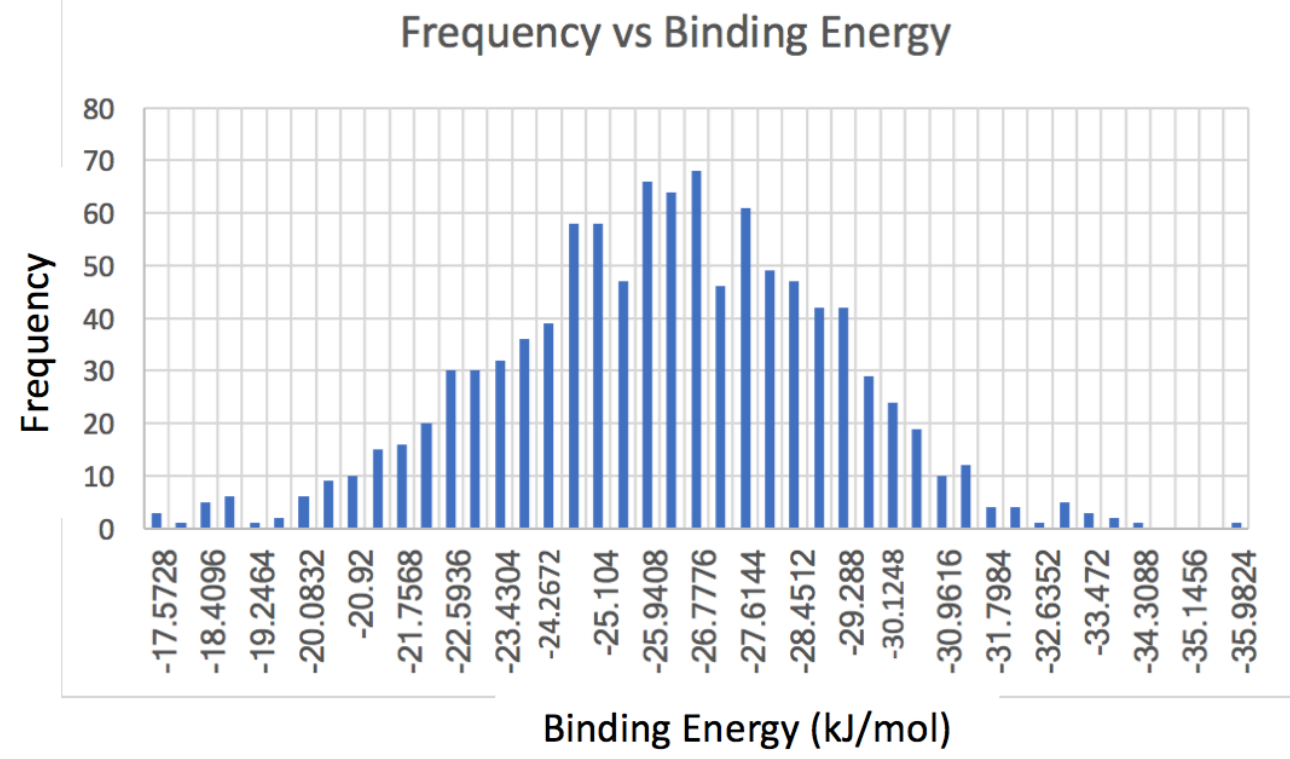

Figure 6 The graph shows the frequency of each predicted binding energy for the 1025 ZINC molecules docked onto the SARS RdRp in Autodock Vina (only the lowest predicted binding energy was considered for each molecule). Molecules with a predicted binding energy of $-33.8904 \mathrm{~kJ} / \mathrm{mol}$ or less were proposed as potential drugs. 


\begin{tabular}{|l|r|}
\hline Molecule & Binding Energy $(\mathrm{kJ} / \mathrm{mol})$ \\
\hline new1 & -24.2672 \\
\hline new2 & -26.3592 \\
\hline new3 & -20.0832 \\
\hline new4 & -24.6856 \\
\hline new5 & -25.5224 \\
\hline new6 & -23.4304 \\
\hline new7 & -23.4304 \\
\hline new8 & -26.7776 \\
\hline new9 & -23.4304 \\
\hline new10 & -29.288 \\
\hline Favipiravir & -25.5224 \\
\hline Galidesivir & -27.6144 \\
\hline Remdesivir & -27.6144 \\
\hline
\end{tabular}

Figure 7 The table shows the lowest predicted binding energy for 10 conditional molecules after being docked onto the SARS RdRp in Autodock Vina. The 10 molecules were generated by the SSVAE with a target $y$ value of -0.4 (equivalent to an IC50 of $1.7 \mathrm{nM}$ ). Below are the lowest predicted binding energies of the 3 comparison groups, compounds favipiravir, galidesivir, and remdesivir, after being docked onto the SARS RdRp in Autodock Vina.

The chemical structures of the top four inhibitors from the ZINC database and one new molecule are shown in Figures 8 and 9, respectively.
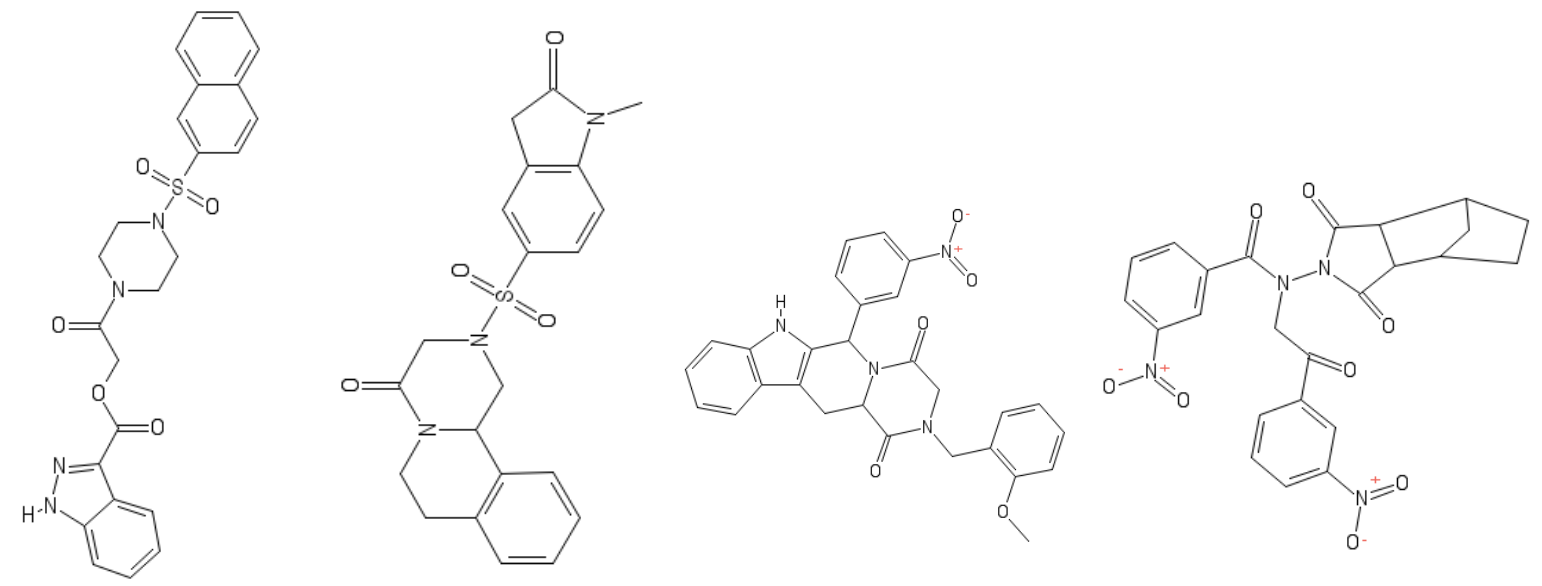

Figure 8 Chemical structure of top four inhibitors for RdRp of SARS-CoV from ZINC database. 


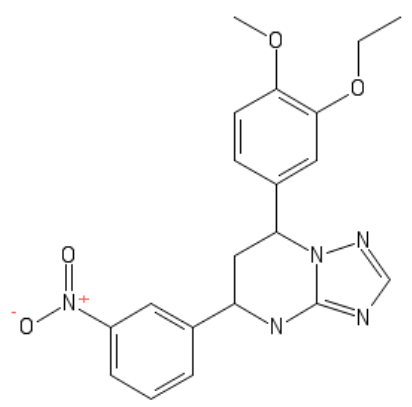

Figure 9 Chemical structure of the SSVAE generated compound with best SARS-CoV RdRp inhibitor properties.

3.4 Autodock Vina Docking on COVID-19 Following the discovery of the RdRp structure of COVID-19 (Gao et. al., 2020), the top 1025 molecules from the ZINC database, the 10 conditionally generated molecules from the SSVAE, and the 3 comparison groups were docked onto the COVID-19 RdRp using Autodock Vina. The lowest predicted binding energy for each molecule was recorded. As seen in Figures 10 and 11, the predicted binding energies for the compounds were lower when binding to the COVID-19 RdRp than when binding to SARS-CoV. The top four molecules from the ZINC dataset when binding to COVID-19, were different from the top four when binding to SARS-CoV. They had binding energies less than or equal to -36.8192 $\mathrm{kJ} / \mathrm{mol}$ and are proposed as potential drugs. Their structures are shown in Figure 12. The most stable compound out of the 10 conditionally generated compounds remained the same when binding to COVID-19 and is proposed as a potential drug. All proposed drugs for COVID-19 had binding energies less than the binding energies of all three comparison groups, indicating stable binding. 
COVID binding energy vs SARS binding energy

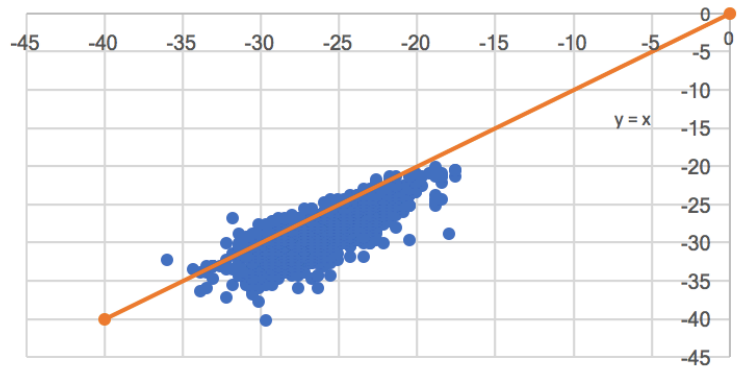

Figure 10 The graph shows the lowest predicted binding energy of the top 1025 ZINC compounds when binding to the SARS-CoV RdRp on the $x$ axis and the lowest predicted binding energy when binding to the COVID-19 RdRp on the $y$ axis. The line $y=x$ is graphed as well. Most points lie below the line, indicating that overall, the COVID-19 binding energies were lower than the SARSCoV energies.

COVID binding energy vs SARS binding energy

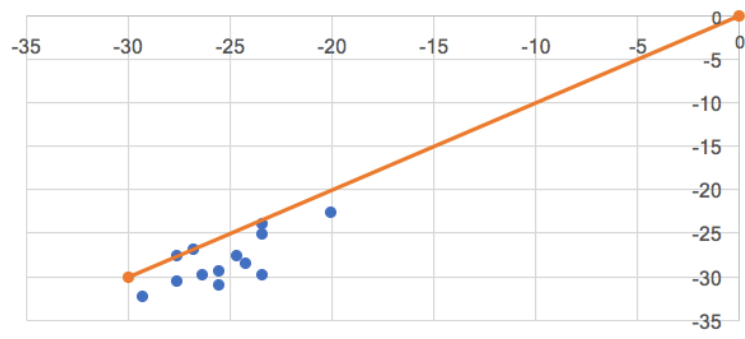

Figure 11 The graph shows the lowest predicted binding energy of the 10 conditionally generated compounds and 3 comparison groups when binding to the SARS-CoVRdRp on the $x$ axis and the lowest predicted binding energy when binding to the COVID-19 RdRp on the $y$ axis. The line $y=x$ is graphed. Most points lie below the line, indicating that overall, the COVID-19 binding energies were lower than the SARS-CoV energies. 

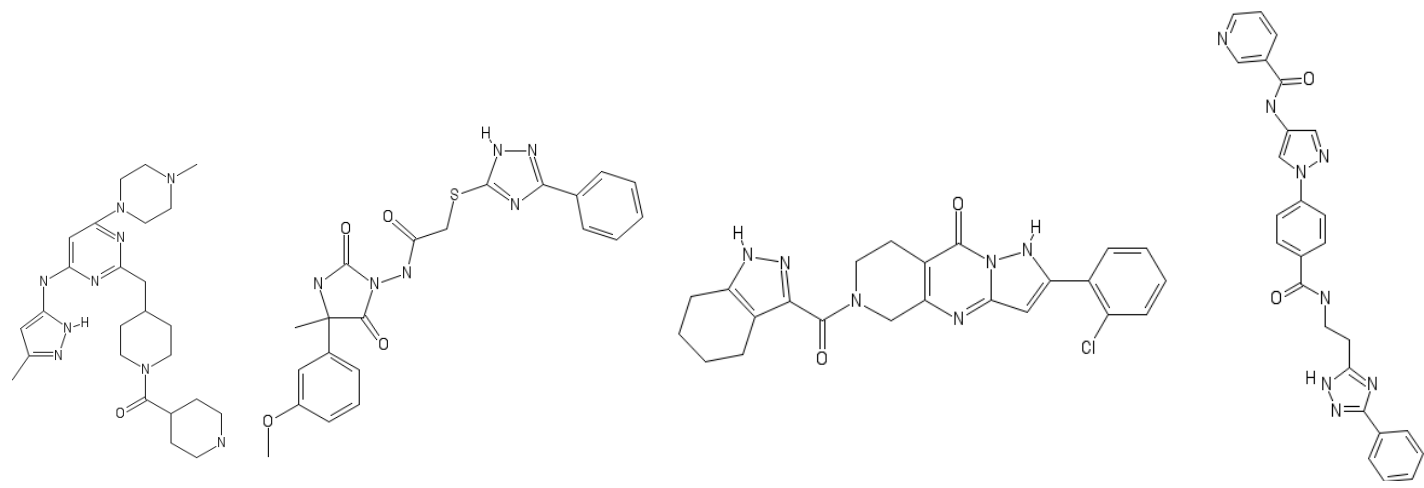

Figure 12 Chemical structure of top four inhibitors for RdRp of COVID-19 from ZINC database.

\section{Conclusion}

There is an urgent need to repurpose or design new drug molecules for COVID-19. Two deep learning-based models from literature are applied to this problem. First, a predictive LSTM model that uses SMILES enumeration was trained. Screening 310k drug-like compounds from the ZINC database led to identification of potential compounds that could be experimentally tested for COVID-19. Each of the compounds showed better binding properties in docking simulations relative to a comparison group of remdesivir, favipiravir, and galidesivir. Additionally, the 310k ZINC database molecules with their predicted IC50s were used to train a generative SemiSupervised Variational AutoEncoder (SSVAE) model. This model was used to generate 10 new molecules by sampling from the latent space to demonstrate its utility. Among the ten new molecules generated by the SSVAE model, the most stable new molecule had binding energy lower than the comparison group of prior drugs. These molecules could potentially be good drug candidates for the SARS CoV and COVID-19. These results show that deep learning-based models could be useful in screening existing compound and generating new molecules to find drugs for COVID-19.

Sources of error can arise from several factors. The LSTM model was trained using limited data which could have resulted in inaccuracies in the parameters. This could have led to further inaccuracies in the SSVAE as the data generated by the LSTM was used to train the generative model. Though an attempt was made to combat this by enumerating the SMILES sequences, in the future, one can perform multiple data augmentation techniques to further improve training. Additionally, the conformations of the ligands that are output in Autodock Vina are based on algorithms that cannot predict the exact binding of the compounds to the protein. These 
conformations affect the predicted binding energy because the placement of the ligand determines what structures come in close proximity of certain atoms on the protein, and therefore what interactions are taking place. By predicting an incorrect conformation, the compound may have a predicted binding energy that is inaccurate. One can counter this by running more dockings in Autodock Vina, however this proved to be impractical in the presented study. Each trial was very time consuming and for this reason, ranges of energies and multiple configurations were considered rather than multiple trials being run. A future study can involve running more trials. Also, more than one docking program can be used to run the same dockings and the results can then be compared.

Further testing would involve using molecular dynamics simulations. Additional future studies can involve experimentally testing top candidates from screened compounds to determine whether they can in fact bind properly to the COVID-19 RdRp. One can also generate more than 10 compounds using the SSVAE, perhaps with a different target y value.

\section{Acknowledgments}

The author thanks Dr. Pathum Weerawarna from Northwestern University for his guidance on biochemistry and using docking software. The author also thanks Ms. Palffy and Ms. Greenberg from Adlai E. Stevenson High School for their support as science fair mentors.

\section{Supplementary Material}

The file named RdRp_Ligand_Data.csv has IC50 data for RpRp binding molecules, which are obtained from the protein databank. The "y" values from LSTM model for 310k drug-like molecules from ZINC database are provided in ZINC_LSTM_y_values.csv. Top 1025 molecules from the ZINC database screened by the LSTM model and 10 new molecules generated by the SSVAE model are given in ZINC_Top1025+New10.xlsx along with their binding energies calculated in AutoDock Vina. 


\section{References}

Bjerrum, E. J. (2017). SMILES Enumeration as Data Augmentation for Neural Network Modeling of Molecules. ArXiv. doi: arXiv:1703.07076v2 [cs.LG]

Centers for Disease Control and Prevention. (2014, March 20). Signs and Symptoms. Retrieved from https://www.cdc.gov/vhf/nipah/symptoms/index.html.

Centers for Disease Control and Prevention. (2019, November 5). Signs and Symptoms. Retrieved December 8, 2019, from https://www.cdc.gov/vhf/ebola/symptoms/index.html.

Centers for Disease Control and Prevention. (n.d.). Zika virus. Retrieved October 11, 2016, from https://www.cdc.gov/zika/healtheffects/index.html

Fuat. (2019, March 25). Google Colab Free GPU Tutorial. Retrieved from https://medium.com/deep-learning-turkey/google-colab-free-gpu-tutorial-e113627b9f5d

GraphPad. (2010, January 19). 50\% of what? How exactly are IC50 and EC50 defined?

Retrieved February 16, 2020, from https:/www.graphpad.com/support/faq/50-of-what-howexactly-are-ic50-and-ec50-defined/

Kang, S., \& Cho, K. (2019). Conditional Molecular Design with Deep Generative Models. Journal of Chemical Information and Modeling. doi: 10.1021/acs.jcim.8b00263

Keras. (n.d.). Keras: The Python Deep Learning library. Retrieved from https://keras.io/

Li, G., \& Clercq, E. D. (2020). Therapeutic options for the 2019 novel coronavirus (2019-nCoV). Nature Reviews Drug Discovery, 19(3), 149-150. doi: 10.1038/d41573-020-00016-0

Morris, G. M. (n.d.). AutoDock. Retrieved November 26, 2017, from http://autodock.scripps.edu/resources/adt

Nguyen, M. (2019, July 10). Illustrated Guide to LSTM's and GRU's: A step by step explanation. Retrieved from https://towardsdatascience.com/illustrated-guide-to-lstms-and-gru-s-a-stepby-step-explanation-44e9eb85bf21

Olah, C. (2015, August 27). Understanding LSTM Networks. Retrieved from https://colah.github.io/posts/2015-08-Understanding-LSTMs/

Raschka, S. (2014, June 26). Molecular docking, estimating free energies of binding, and AutoDock's semi-empirical force field. Retrieved February 17, 2018, from http://sebastianraschka.com/Articles/2014 autodock energycomps.html

Sterling, T., \& Irwin, J. J. (2015). ZINC 15 - Ligand Discovery for Everyone. Journal of Chemical Information and Modeling, 55(11), 2324-2337. doi: 10.1021/acs.jcim.5b00559 
Tchesnokov, E., Feng, J., Porter, D., \& Götte, M. (2019). Mechanism of Inhibition of Ebola Virus RNA-Dependent RNA Polymerase by Remdesivir. Viruses, 11(4), 326. doi: $10.3390 / \mathrm{v} 11040326$

TensorFlow. (n.d.). TensorFlow. Retrieved from https://www.tensorflow.org/

Vasudev. (2019, October 25). What is One Hot Encoding? Why And When do you have to use it? Retrieved from https://hackernoon.com/what-is-one-hot-encoding-why-and-when-do-youhave-to-use-it-e3c6186d008f

Venkataraman, S., Prasad, B., \& Selvarajan, R. (2018). RNA Dependent RNA Polymerases: Insights from Structure, Function and Evolution. Viruses, 10(2), 76. doi: 10.3390/v10020076

Wan, Y., \& Chatterjee, K. (2018, July 13). RNA. Retrieved December 8, 2019, from https://www.britannica.com/science/RNA. 